\title{
Adaptive WHT Aided QAM for Fading Channels Subjected to Impulsive Noise
}

\author{
Byoungjo Choi, Member, IEEE, and Lajos Hanzo, Fellow, IEEE
}

\begin{abstract}
The Bit Error Rate (BER) expressions of WalshHadamard Transform (WHT) aided Quadrature Amplitude Modulation (QAM) transmitted in impulsive noise environments are derived. It is found that there is a crossover point between the conventional and WHT-aided schemes' BER curves, above which WHT is beneficial in terms of the corresponding BER performance. The exact crossover SNR is analysed and its approximate closed-form expression is derived for a QPSK scheme based on the Chernoff bound. An adaptive WHT scheme is proposed for QAM systems, where the transmitter activates the WHT only when the instantaneous SNR at the receiver is higher than the crossover SNR. The theoretical BER of our adaptive WHT scheme is derived for QAM systems operating over Nakagami-m fading channels. It is found that our adaptive WHT scheme is effective in terms of mitigating the detrimental effects of impulsive noise over the entire SNR range.
\end{abstract}

Index Terms-WHT, impulsive noise, adaptive, QAM.

\section{QAM SySTEMS IN IMPULSIVE NOISE}

$\mathbf{T}$ HE noise experienced in the communications scenarios exemplified in [1]-[3] is non-Gaussian, since it has a strong impulsive component. Middleton's Class-A interference model has been widely used for investigating the performance of various communication systems operating in environments subjected to impulsive noise [1]. It has also been recognised that a truncated version of the Class-A interference model provides a tractable alternative description of the impulsive noise [2]. In this two-component Gaussian mixture model, a discrete-time complex-valued impulsive noise sample $n$ is assumed to have the following probability density function [2]:

$$
f_{n}(n)=\frac{\lambda_{0}}{\pi \sigma^{2}} \exp \left(-\frac{|n|^{2}}{\sigma^{2}}\right)+\frac{\lambda_{1}}{\pi \kappa \sigma^{2}} \exp \left(-\frac{|n|^{2}}{\kappa \sigma^{2}}\right),
$$

where the first term corresponds to a nominal background noise having the variance of $\sigma^{2}$, while the second term to a less-frequent heavy-tail impulse noise with the variance of $\kappa \sigma^{2}$. The frequency of the impulsive term is controlled by $\lambda_{1}$ and it is related to $\lambda_{0}$ as $\lambda_{0}+\lambda_{1}=1$. The typical ranges of $\lambda_{1}$ and $\kappa$ are $10^{-2} \sim 0.5$ and $10^{2} \sim 10^{4}$, respectively [1], [2].

In a QAM system operating in a channel contaminated by impulsive noise, the received complex low-pass equivalent discrete time sample $r$ can be expressed as

$$
r=s+n,
$$

Manuscript received March 25, 2013. The associate editor coordinating the review of this letter and approving it for publication was R. Souza.

The financial support of University of Incheon, and that of the European Research Council's Advanced Fellow Grant is gratefully acknowledged.

B. Choi is with the Department of Embedded Systems Engineering, University of Incheon, Republic of Korea.

L. Hanzo is with the Department of Electronics and Computer Science, University of Southampton, UK (e-mail: 1h@ecs.soton.ac.uk).

Digital Object Identifier 10.1109/LCOMM.2013.13.130690 where $s$ is a transmitted QAM symbol and $n$ is an additive impulsive noise sample. The exact BER of Gray-coded square QAM constellations can be expressed as [4]

$$
P_{b}(\gamma)=\sum_{i} A_{i}\left\{\lambda_{0} Q\left(\sqrt{a_{i} \gamma}\right)+\lambda_{1} Q\left(\sqrt{a_{i} \gamma / \kappa}\right)\right\},
$$

where $\gamma$ is the SNR considering the background noise only, which is defined as $\gamma \triangleq E\left[\left|s_{k}\right|^{2} / \sigma^{2}\right]$, and the set of values for the QAM modulation-order-dependent constants $\left\{A_{i}, a_{i}\right\}$ can be found, for example, in (47) of [5]. Specifically, the set of constants are $\{(1,1)\}$ for QPSK, and $\{(3 / 4,1 / 5),(2 / 4,9 / 5),(-1 / 4,5)\}$ for 16-QAM [5].

\section{WHT-QAM SYSTEMS IN IMPULSIVE NOISE}

In WHT-aided QAM systems, $N$ number of consecutive QAM symbols are grouped together and then the WHT is applied before their transmission [3], [6]. A WHT-aided QAM system is equivalent to a fully-loaded Walsh-spread QAMmodulated Code Division Multiple Access (CDMA) system. However, unlike conventional CDMA systems, applying WHT does not incur any bandwidth expansion, because $N$ consecutive symbol durations are used for transmitting $N$ WHTassisted QAM symbols. The received symbols of our WHTaided QAM system can be represented as

$$
\mathbf{r}=\mathbf{W}_{N} \mathbf{s}+\mathbf{n},
$$

where $\mathbf{r}, \mathbf{s}$, and $\mathbf{n}$ are the $N \times 1$ vector of received symbols, transmitted QAM symbols and impulsive noise samples. The $N \times N$ matrix $\mathbf{W}_{N}$ in (4) is the normalized Walsh-Hadamard matrix satisfying $\mathbf{W}_{N} \mathbf{W}_{N}=\mathbf{I}_{N}$. In order to detect the transmitted QAM symbols, the receiver applies WHT to the received symbols given in (4), resulting in

$$
\hat{\mathbf{s}}=\mathbf{W}_{N} \mathbf{r}=\mathbf{s}+\mathbf{W}_{N} \mathbf{n}=\mathbf{s}+\tilde{\mathbf{n}} .
$$

Noting that the elements of rows in $\mathbf{W}_{N}$ are \pm 1 , we can see that an element $\tilde{n}$ in the vector $\tilde{\mathbf{n}}$ is simply a sum of positive or negative values of $\left\{n_{i}\right\}$. Assuming that $N$ number of noise samples $n_{i}$ in the vector $\mathbf{n}$ are independent and identically distributed as in (1), the PDF of a scalar $\tilde{n}$ in $\tilde{\mathbf{n}}$ can be expressed in terms of $(N+1)$ number of Gaussian mixture distributions, which is formulated as [2], [4]

$$
\begin{aligned}
f_{\tilde{n}}(\tilde{n}) & =\prod_{k=0}^{N-1} f_{n}(\tilde{n})=\left\{f_{n}(\tilde{n})\right\}^{N} \\
& =\sum_{k=0}^{N} p_{k} \frac{1}{\pi v_{k} \sigma^{2}} \exp \left(-\frac{|\tilde{n}|^{2}}{v_{k} \sigma^{2}}\right),
\end{aligned}
$$




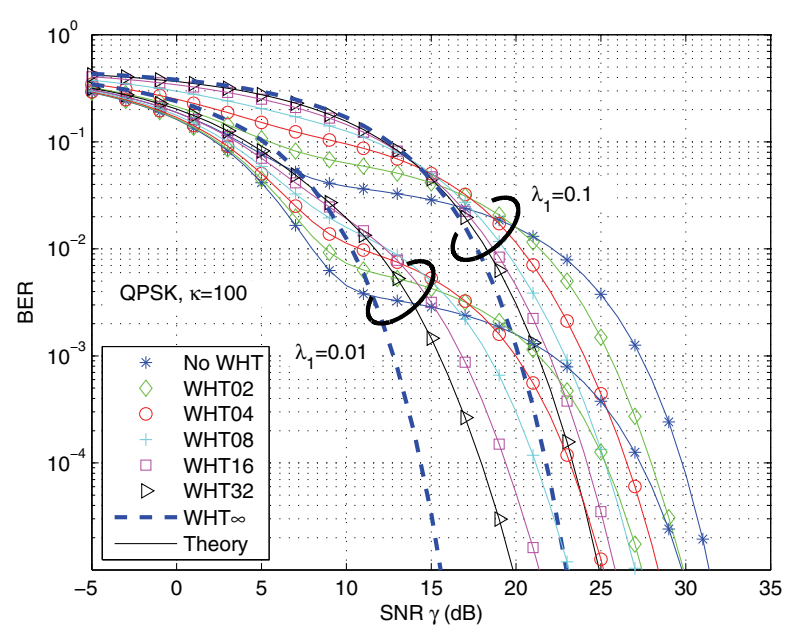

Fig. 1. The BER of WHT-aided QPSK for transmission over channels contaminated by impulsive noise.

where $p_{k}$ is the probability of the $k$-th Gaussian term with a variance of $v_{k} \sigma^{2}$. The probability $p_{k}$ is given by [2], [4]

$$
p_{k}=\frac{N !}{(N-k) ! \times k !} \lambda_{0}^{k} \lambda_{1}^{N-k}
$$

and the variance scaling factor $v_{k}$ is given as [2], [4]

$$
v_{k}=\frac{k+\kappa(N-k)}{N} \text {. }
$$

Since the noise distribution in (6) is comprised of a sum of Gaussian PDFs, the BER of our WHT-aided QAM scheme may be expressed as

$$
P_{b}(\gamma ; N)=\sum_{i} \sum_{k=0}^{N} A_{i} p_{k} Q\left(\sqrt{a_{i} \gamma / v_{k}}\right) .
$$

The BER analysis of (6)-(9) constitutes an extension of the results generated for the BER in the context of a singleuser BPSK CDMA system in [2] and for the Symbol Error Rate (SER) in the context of a multicarrier QAM system in [4] operating in an impulsive channel. When the WHT order obeys $N \rightarrow \infty$, the central limit theorem states that the noise distribution of $\tilde{n}$ becomes Gaussian with the variance of $\left(\lambda_{0}+\lambda_{1} \kappa\right) \sigma^{2}$ [2]. In this scenario, the BER of (9) simply converges to

$$
P_{b}(\gamma ; \infty)=\sum_{i} A_{i} Q\left(\sqrt{a_{i} \gamma /\left(\lambda_{0}+\lambda_{1} \kappa\right)}\right) .
$$

The BERs of (9) and (10) derived for WHT-aided QPSK systems are depicted in Fig. 1 for various values of the WHT order $N$, when the power ratio of the impulsive noise term to the background term is $\kappa=100$ and the probability of impulsive noise contamination is $\lambda_{1}=0.1$ or $\lambda_{1}=0.01$ [2]. It can be seen in Fig. 1 that the BER curve of a QPSK system operating without WHT exhibits a characteristic plateau, when the BER is roughly $0.5 \lambda_{1}$, where the increase in SNR results in only a slight decrease in the corresponding BER. The WHT employed effectively spreads the less frequent high-magnitude noise samples over several symbols in order to achieve an averaging effect. As observed in Fig. 1, the averaging effect of

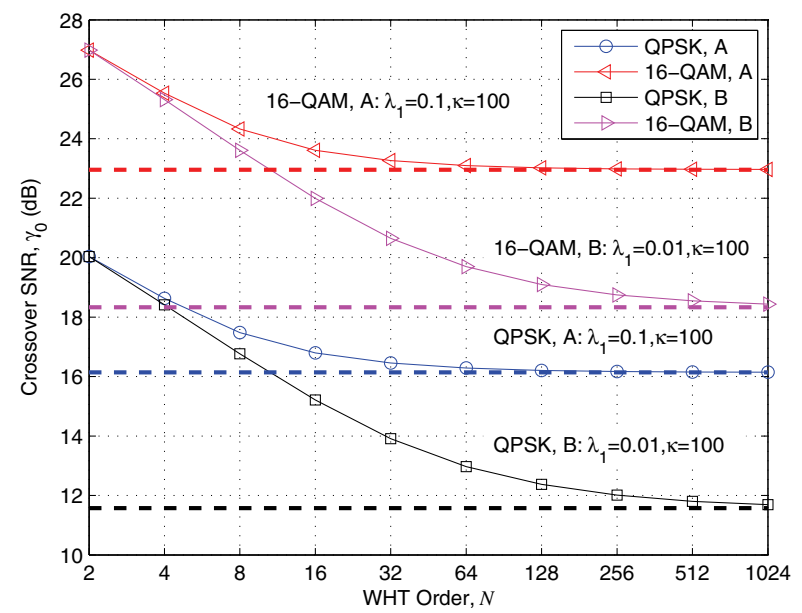

Fig. 2. The crossover SNR values in $\mathrm{dB}$ for WHT-aided QAM for the various values of WHT order $N$ over impulse-noise-contaminated channels

WHT considerably improves the BER for SNR values higher than $16 \mathrm{~dB}$ when $\lambda_{1}=0.1$. However, it can also be observed that it adversely affects the BER in the low-SNR region. Based on these observations, we propose an adaptive WHT scheme for QAM systems operating in fading channels subjected to impulsive noise.

\section{AdAPTIVE WHT-AIDED QAM}

\section{A. Crossover SNR}

In the proposed adaptive WHT-aided QAM scheme operating in a narrow-band fading channel, the receiver makes a decision whether WHT will be beneficial or not for improving its BER performance, based on the instantaneous SNR, and feeds the decision back to the transmitter. Here, we introduce the crossover SNR $\gamma_{0}$ as the boundary, where the BER of WHT-aided QAM given in (9) becomes equal to the BER of conventional QAM without WHT given in (3). Specifically, the following relationship holds at the crossover SNR $\gamma_{0}$;

$$
P_{b}\left(\gamma_{0} ; N\right)=P_{b}\left(\gamma_{0}\right) .
$$

Since we have expressed both the BERs in (11) as closed form expressions, we can readily obtain the values of $\gamma_{0}$ for the various values of the WHT order $N$ using a numerical method.

Fig. 2 depicts the crossover SNR values for QPSK and 16QAM systems for the impulsive noise parameter sets of A and $\mathrm{B}$, which converge to $\gamma_{0, \infty}$ values (dashed lines) as the WHT order $N$ increases. This asymptotic crossover SNR $\gamma_{0, \infty}$ can be found by equating (10) and (3) for QPSK, yielding

$$
\lambda_{0} Q\left(\sqrt{\gamma_{0, \infty}}\right)+\lambda_{1} Q\left(\sqrt{\frac{\gamma_{0, \infty}}{\kappa}}\right)=Q\left(\sqrt{\frac{\gamma_{0, \infty}}{\lambda_{0}+\lambda_{1} \kappa}}\right) .
$$

An approximate solution of (12) can be obtained by using the Chernoff bound of the $Q$-function, $Q(x) \simeq 0.5 \exp \left(-x^{2} / 2\right)$, which can be expressed as

$$
\gamma_{0, \infty} \cong 2\left(\ln \lambda_{1}^{-1}\right) \frac{\kappa\left(\lambda_{0}+\lambda_{1} \kappa\right)}{\lambda_{0}(\kappa-1)} .
$$


When $\kappa \gg 10$ and $\lambda_{1} \kappa \gg 1,(13)$ can be further approximated as

$$
\gamma_{0, \infty} \cong 2\left(\ln \lambda_{1}^{-1}\right) \lambda_{1} \kappa .
$$

For example, when $\lambda_{1}=0.1$ and $\kappa=100$, the approximate closed form solution of (14) gives $16.63 \mathrm{~dB}$, which is close to the actual value of $16.14 \mathrm{~dB}$ obtained by numerically solving (12). Expressing the crossover SNR of (14) in $\mathrm{dB}$, we have

$$
\gamma_{0, \infty}[d B]=q_{d B}+\Gamma_{d B}+6.6325,
$$

where $q_{d B}=10 \log (q)$ when $\lambda_{1}=10^{-q}$ and $\Gamma_{d B}=$ $10 \log \left(\lambda_{1} \kappa\right)$. In Middleton's Class-A impulse noise model, $\lambda_{1}$ corresponds to the impulsive index $A$ and $\lambda_{1} \kappa$ corresponds to $\Gamma^{\prime}$, which is the ratio of the power in the Gaussian portion of the noise to the power in the Poisson component [1]. The approximate crossover SNRs for 16-QAM systems can be obtained by adding $7 \mathrm{~dB}$ to those for QPSK, considering that 16-QAM requires five times more power than QPSK while maintaining the same minimum distance between the legitimate constellation points. The crossover SNR expressed in (15) gives an insight regarding the effective operating SNR region of our WHT-aided system in terms of the impulsive noise model parameters.

\section{B. The Average BER of Adaptive WHT-aided QAM}

In a fading scenario the SNR $\gamma$ in (3) and (9) is no longer a constant, but a random variable with a PDF of $f_{\gamma}(\gamma ; \bar{\gamma})$ having a mean value of $\bar{\gamma}$ [5]. Assuming a perfect SNR estimation and an ideal feedback from the receiver regarding the activation or deactivation of the WHT, the average BER of our adaptive WHT-aided QAM scheme may be expressed as

$$
\bar{P}_{b}\left(\bar{\gamma} ; \gamma_{0}\right)=\int_{0}^{\gamma_{0}} P_{b}(\gamma) f_{\gamma}(\gamma ; \bar{\gamma}) d \gamma+\int_{\gamma_{0}}^{\infty} P_{b}(\gamma ; N) f_{\gamma}(\gamma ; \bar{\gamma}) d \gamma
$$

where the crossover SNR $\gamma_{0}$ satisfies (11), while the BER $P_{b}(\gamma)$ of conventional QAM without WHT is given in (3), and the BER of WHT-aided QAM $P_{b}(\gamma ; N)$ is given in (9).

Fading channels are often modeled as Nakagami-fading channels. The PDF of the instantaneous channel SNR $\gamma$ when communicating over a Nakagami-fading channel is given as

$$
f_{\gamma}(\gamma ; \bar{\gamma})=\left(\frac{m}{\bar{\gamma}}\right)^{m} \frac{\gamma^{m-1}}{\Gamma(m)} \exp \left(-m \frac{\gamma}{\bar{\gamma}}\right), \gamma \geq 0
$$

where the parameter $m$ governs the severity of fading covering a wide range of fading channels, such as Rayleigh fading, Rician-like fading and near Gaussian channels [5]. Since the BER expressions of $P_{b}(\gamma)$ and $P_{b}(\gamma ; N)$ used in (16) are given by a weighted sum of $Q(\sqrt{a \gamma})$-function terms with appropriate constants $a$, it is sufficient to calculate the following regional integral for expressing the BER of (16):

$$
\begin{aligned}
P_{r}(\alpha, \beta ; \bar{\gamma}, a) & =\int_{\alpha}^{\beta} Q(\sqrt{a \gamma}) f_{\gamma}(\gamma ; \bar{\gamma}) d \gamma \\
& =F(\gamma ; \bar{\gamma}, a)]_{\alpha}^{\beta},
\end{aligned}
$$

where $F(\gamma ; \bar{\gamma}, a)$ is an indefinite integral formulated as

$$
F(\gamma ; \bar{\gamma}, a) \triangleq \int Q(\sqrt{a \gamma}) f_{\gamma}(\gamma ; \bar{\gamma}) d \gamma
$$

The solution of (20) for the integer values of $m$ is given in [5, Appendix B], which can be expressed in a more compact alternative form as

$$
F(\gamma ; \bar{\gamma}, a)=\sum_{d=0}^{m-1} \frac{(m / \bar{\gamma})^{d}}{\Gamma(d+1)}\left\{F_{1}(\gamma ; \bar{\gamma}, d, a)+F_{2}(\gamma ; \bar{\gamma}, d, a)\right\},
$$

where the component indefinite integrals $F_{1}(\cdot)$ and $F_{2}(\cdot)$ are given as

$$
\begin{aligned}
& F_{1}(\gamma ; \bar{\gamma}, d, a)=-\gamma^{d} Q(\sqrt{a \gamma}) \exp (-m \gamma / \bar{\gamma}), \\
& F_{2}(\gamma ; \bar{\gamma}, d, a)=\frac{1}{2} \sqrt{\frac{a}{2 \pi}} \mu^{(d+1 / 2)} \Gamma(d+0.5, \gamma / \mu) .
\end{aligned}
$$

The function $\Gamma(s, x)$ in (23) is the upper incomplete-Gamma function defined as [7]

$$
\Gamma(s, x) \triangleq \int_{x}^{\infty} t^{s-1} e^{-t} d t .
$$

The SNR-dependent constant $\mu$ in (23) is given as

$$
\mu=2 \bar{\gamma} /(2 m+a \bar{\gamma}),
$$

where, again, $m$ is the Nakagami parameter and $a$ is a QAMorder-dependent constant.

When the Nakagami fading parameter $m$ is not an integer, it remains an open challenge to derive the closed form solution of (20). However, using the analytically tractable approximation of the Gaussian Q-function provided in [8], the regional BER of (18) can be expressed as in the Appendix, yielding

$$
\begin{aligned}
P_{r}(\alpha, \beta ; \bar{\gamma}, a) \simeq \sum_{n=1}^{n a} K_{n}\left\{\Gamma\left(\frac{2 m+n-1}{2}, \frac{\alpha}{\mu}\right)\right. \\
\left.-\Gamma\left(\frac{2 m+n-1}{2}, \frac{\beta}{\mu}\right)\right\},
\end{aligned}
$$

where $n a$ is the number of terms used in the series for approximating the Gaussian Q-function [8] and the constants $K_{n}$ are given by (34) of the Appendix.

The average BER of our adaptive WHT-aided QAM systems of (16) can then be expressed with the aid of (18) or (26) as

$$
\begin{aligned}
\bar{P}_{b}\left(\bar{\gamma} ; \gamma_{0}\right)= & \sum_{i} A_{i}\left\{\lambda_{0} P_{r}\left(0, \gamma_{0} ; \bar{\gamma}, a_{i}\right)+\lambda_{1} P_{r}\left(0, \gamma_{0} ; \bar{\gamma}, a_{i} / \kappa\right)\right\} \\
& +\sum_{i} \sum_{k=0}^{N} A_{i} p_{k} P_{r}\left(\gamma_{0}, \infty ; \bar{\gamma}, a_{i} / v_{k}\right) .
\end{aligned}
$$

In general, the higher the number of terms, $n a$, in (26), the more accurate the approximated regional BER expression of (26) becomes. Our numerical investigations revealed that a value of $n a=15$ is sufficient for arriving at an average BER of $10^{-3}$ within $0.05 \mathrm{~dB}$ from the exact value obtained by numerical integration.

In conclusion, the BERs of various adaptive WHT-aided QPSK schemes are depicted in Fig. 3 for the two different scenarios, when the WHT order is $N=128$. Case A corresponds to a severely faded impulsive noise environment when $\lambda_{1}=0.1$ and $\kappa=100$ with the Nakagami fading parameter value of $m=1$. By contrast, Case B corresponds to a relatively benign environment using the parameters of $\lambda_{1}=0.01, \kappa=100$, and $m=15.5$. The BER of the adaptive WHT-aided 16-QAM scheme is also depicted in Fig. 3 for 


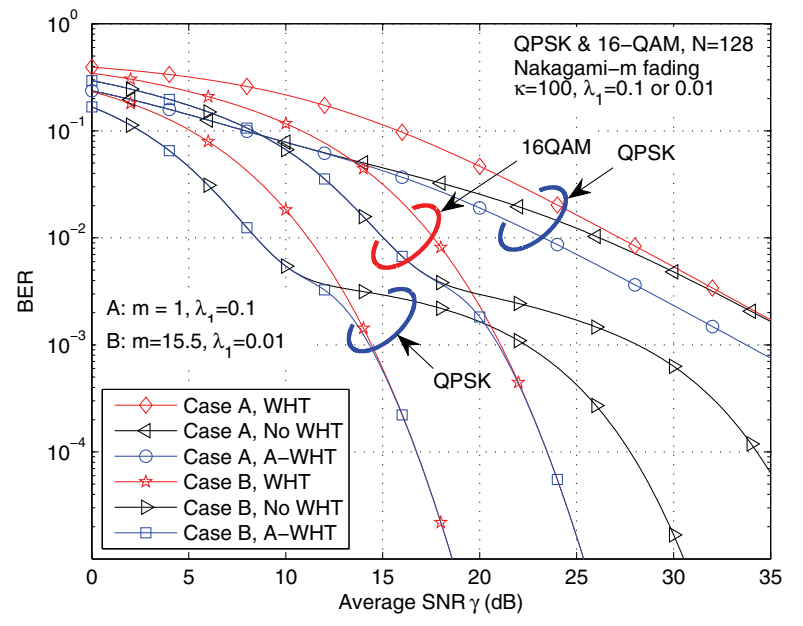

Fig. 3. The average BER of adaptive WHT-aided QAM over Nakagami$m$ fading channel subjected to impulsive noise when $m=1,\left[\lambda_{1}=0.1\right.$, $\kappa=100]$ for Case A, and $m=15.5,\left[\lambda_{1}=0.01, \kappa=100\right]$ for Case B.

Case B, which approximately corresponds to a $7 \mathrm{~dB}$-shifted QPSK BER curve. Observe that our adaptive WHT-aided QAM system outperforms both the conventional QAM system without WHT and the non-adaptive WHT-aided system always activating the WHT across the entire SNR range.

The relative SNR gain of the proposed adaptive WHT scheme over the non-adaptive WHT scheme recorded at high SNRs becomes more pronounced, when the Nakagami fading parameter $m$ is low, because the instantaneous SNR value varies substantially. Hence our regime leads to a better exploitation of the channel quality by the adaptive scheme. Observe in Fig. 3 that this SNR gain observed for our Rayleigh fading scenario of $m=1$ is approximately $3 \mathrm{~dB}$ even when the SNR is higher than $30 \mathrm{~dB}$. When the Nakagami fading parameter is $m=15.5$, the SNR gain of our adaptive WHT scheme over the permanent-spreading aided WHT scheme diminishes at high SNRs, but it provides up to $7 \mathrm{~dB}$ gain at low SNRs.

The main drawback of applying WHT to QAM symbols is an increase in its Peak-to-Average Power Ratio (PAPR) values. The family of PAPR reduction schemes developed for OFDM may be invoked for reducing the PAPRs in WHT-aided QAM systems.

\section{APPENDIX}

\section{Derivation OF APPROXIMATE REgIONAL BER IN (26)}

The Gaussian Q-function term $Q(\sqrt{a \gamma})$ in (18) may be approximated as [8]

$$
Q(a \gamma) \simeq e^{-\frac{a \gamma}{2}} \sum_{n=1}^{n a} c_{n}(a \gamma)^{\frac{n-1}{2}} .
$$

The coefficient $c_{n}$ in (28) is given in [8] as

$$
c_{n}=\frac{(-1)^{n+1} A^{n}}{B \sqrt{\pi} 2^{(n+1) / 2} n !},
$$

where the constants $A$ and $B$ are given in [9] as $A=1.98$ and $B=1.135$.
The regional integral $P_{r}(\alpha, \beta ; \bar{\gamma}, a)$ of (18) may then be expressed with the aid of (28), as

$$
\begin{aligned}
P_{r} & \simeq \sum_{n=1}^{n a} \int_{\alpha}^{\beta} c_{n} e^{-\frac{a \gamma}{2}}(a \gamma)^{\frac{n-1}{2}}\left(\frac{m}{\bar{\gamma}}\right)^{m} \frac{\gamma^{m-1}}{\Gamma(m)} e^{-m \frac{\gamma}{\gamma}} d \gamma \\
& =\sum_{n=1}^{n a} K_{n}^{\prime} \int_{\alpha}^{\beta} \gamma^{m+\frac{n}{2}-\frac{3}{2}} e^{-\frac{\gamma}{\mu}} d \gamma
\end{aligned}
$$

where the constant $K_{n}^{\prime}$ is defined as

$$
K_{n}^{\prime} \triangleq c_{n} a^{\frac{n-1}{2}}\left(\frac{m}{\bar{\gamma}}\right)^{m} \frac{1}{\Gamma(m)}
$$

and $\mu$ is given as $\mu=2 \bar{\gamma} /(2 m+a \bar{\gamma})$. Applying the change of variable as $t=\gamma / \mu$ and defining $s=m+n / 2-1 / 2$, the last integral term of (30) may be expressed as

$$
\begin{aligned}
\int_{\alpha}^{\beta} \gamma^{s-1} e^{-\frac{\gamma}{\mu}} d \gamma & =\int_{\alpha / \mu}^{\beta / \mu} \mu^{s} t^{s-1} e^{-t} d t \\
& =\mu^{s}\{\Gamma(s, \alpha / \mu)-\Gamma(s, \beta / \mu)\},
\end{aligned}
$$

where $\Gamma(s, x)$ is the upper incomplete-Gamma function defined as [7]

$$
\Gamma(s, x) \triangleq \int_{x}^{\infty} t^{s-1} e^{-t} d t .
$$

Substituting(32) into (30) and defining a new constant $K_{n}$ as

$$
\begin{aligned}
K_{n} & =K_{n}^{\prime} \mu^{m+n / 2-1 / 2} \\
& =c_{n} a^{(n-1) / 2}\left(\frac{m}{\bar{\gamma}}\right)^{m} \frac{1}{\Gamma(m)} \mu^{m+n / 2-1 / 2},
\end{aligned}
$$

we finally arrive at

$$
\begin{aligned}
P_{r}(\alpha, \beta ; \bar{\gamma}, a) \simeq \sum_{n=1}^{n a} K_{n} & \left\{\Gamma\left(\frac{2 m+n-1}{2}, \frac{\alpha}{\mu}\right)\right. \\
& \left.-\Gamma\left(\frac{2 m+n-1}{2}, \frac{\beta}{\mu}\right)\right\} .
\end{aligned}
$$

\section{REFERENCES}

[1] A. D. Spaulding and D. Middleton, "Optimum reception in an impulsive interference environment-part I: coherent," IEEE Trans. Commun., vol. 25, no. 9, pp. 910-923, Sep. 1977.

[2] B. Aazhang and H. V. Poor, "Performance of DS/SSMA communications in impulsive channels-part I: linear correlation receivers," IEEE Trans. Commun., vol. 35, no. 11, pp. 1179-1188, Nov. 1987.

[3] H. Bogucka, "On the impact of the impulse noise on the WHT/OFDM transmission," IEEE Commun. Lett., vol. 9, no. 1, pp. 37-39, Jan. 2005.

[4] M. Ghosh, "Analysis of the effects of impulse noise on multicarrier and single carrier QAM systems," IEEE Trans. Commun., vol. 44, no. 2, pp. 145-147, Feb. 1996.

[5] B. Choi and L. Hanzo, "Optimum mode-switching-assisted constantpower single- and multicarrier adaptive modulation," IEEE Trans. Veh. Technol., vol. 52, no. 3, pp. 536-560, May 2003.

[6] L. Hanzo et al., OFDM and MC-CDMA for Broadband Multi-User Communications, WLANs and Broadcasting. Wiley-IEEE Press, 2003.

[7] I. S. Gradshteyn and I. M. Ryzhik, Table of Integrals, Series, and Products. Academic Press, 2007.

[8] Y. Isukapalli and B. D. Rao, "An analytically tractable approximation for the Gaussian Q-function," IEEE Commun. Lett., vol. 12, no. 9, pp. 669-671, Sep. 2008.

[9] G. K. Karagiannidis and A. S. Lioumpas, "An improved approximation for the Gaussian Q-function," IEEE Commun. Lett., vol. 11, no. 8, pp. 644-646, Aug. 2007. 